\title{
¿Cómo Afectó la Pandemia en el Uso y Aplicación de Tecnologías en las Familias de los Estudiantes del Sector de San Juan de Calderón en la Ciudad de Quito?
}

How the Pandemic Affected the Use and Application of Technologies in the Families of Students in the San Juan de Calderón Sector in the City f Quito-Ecuador

\begin{tabular}{c} 
Andrade G. ${ }^{\mathbf{1}}$; Anzules L. ${ }^{2}$ \\
${ }^{1}$ Multiversidad Mundo Real Edgar Morin, Doctorado en Pensamiento Complejo, México D. F. \\
e-mail: guadalupeandrade2001@yahoo.com \\
$\begin{array}{c}{ }^{2} \text { Multiversidad Mundo Real Edgar Morin, Maestría en Investigación Integrativa, México D. F. } \\
\text { e-mail: ladyanzules26@hotmail.com }\end{array}$ \\
\hline $\begin{array}{c}\text { Información del artículo } \\
\text { Recibido: } 23 / 02 / 2021 \\
\text { Aceptado: } 17 / 03 / 2021\end{array}$ DOI https://doi.org/10.29166/ingenio.v4i1.2980
\end{tabular}

\section{RESUMEN}

Considerando que la educación en la actualidad busca desarrollar aprendizajes significativos con el uso adecuado de las TIC para dar la oportunidad a estudiantes y docentes y poder generar clases más dinámicas y atractivas. En el momento que llegó la pandemia al Ecuador, desencadenó una crisis sanitaria que también hizo palpable la gran brecha socioeconómica de todos los países, en especial de nuestro país. Esta dura realidad desencadenó en las familias problemas como altos niveles de estrés, frustración y ansiedad al no poder contar con los recursos necesarios para acceder a la educación, ahora vinculada directamente a recursos como el internet. Esta realidad nos impulsa a la búsqueda de una verdadera solución.

Palabras clave: Educación, TIC, pandemia, pobreza.

\begin{abstract}
Considering that education currently seeks to develop meaningful learning, with the appropriate use of ICT, seeking to give students and teachers the opportunity to generate more dynamic and attractive classes. When the pandemic arrived in Ecuador, it unleashed a health crisis, which also made palpable the great socioeconomic gap in all countries, especially in our country. This harsh reality triggered problems in families such as high levels of stress, frustration and anxiety due to not being able to have the necessary resources to access education, now directly linked to resources such as the internet. This reality drives us to search for a true solution.
\end{abstract}

Keywords: Education, TIC, pandemic, poverty. 


\section{Introducción}

En la actualidad, podemos decir que la educación de este nuevo siglo busca mucho más que la repetición de contenidos, se trata más bien de una construcción de conocimientos de forma crítica a través del desarrollo de las destrezas con criterio de desempeño, donde como un complemento están inmersas las TIC [3], que brinda a las clases diversidad, dinámica y un cambio del esquema de aprendizaje en los estudiantes. En la actualidad, es una herramienta fundamental para fortalecer la calidad educativa de toda la comunidad.

Desgraciadamente, en el sector de San Juan de Calderón y en otros sectores de características vulnerables, viven otra realidad por su situación socioeconómica que implica poca o ninguna accesibilidad a las redes tecnológicas, situación que afecta el desarrollo de sus destrezas en el proceso de enseñanza aprendizaje. Por tal razón, el objetivo de esta investigación es identificar cómo las familias han enfrentado este desafío educativo, con las TIC en tiempos de pandemia

En el Ecuador se puede describir que ha habido un sinnúmero de dificultades durante esta crisis sanitaria, aparte del contagio masivo se pudo detectar otra problemática que la pandemia hizo visible, como el efectivo uso de la tecnología y sus aplicaciones. Las familias, por diversos factores como no tener acceso al internet y el desconocimiento en el uso de las TIC, ha dado como resultado, primero, en el aspecto emocional, estrés en las familias y estudiantes, frustración al no poder conectarse a las clases y enviar sus actividades, también la parte económica, al no poder acceder a un equipo tecnológico que le ayude en su proceso educativo, y otro aspecto importante, el nivel académico de los padres y madres de familia, ya que al ser un sector rural las personas se han dedicado a labores del campo, sin dar verdadera relevancia a su formación escolar, como consecuencia no pueden ayudar a sus hijos a realizar sus tareas e investigaciones, lo que deriva en la deserción escolar de algunos estudiantes [4].

\subsection{Historia de las TIC, en la educación}

En el desarrollo de la sociedad y de la humanidad, los cambios y revoluciones sociales no se pueden relatar sin que la tecnología esté inmersa en ese escenario, solo como ejemplo podemos mencionar los cambios en la comunicación que generó la presencia de la imprenta, la radio, la televisión, el teléfono, el computador, el fax, el localizador o beeper, el celular y la web, con las redes sociales, y toda la información que está al alcance de los estudiantes, profesionales, investigadores y población en general [1].

Se puede decir que desde 1910 la sociedad comenzó a dar grandes saltos, con los avances tecnológicos que facilitaron la vida y la comunicación entre todas las personas. Y después se fue implementando en el campo laboral y en la educación. Todo está relacionado, vinculado como una red neuronal que conecta los logros del pasado con los del presente y los del futuro. Históricamente, nunca hemos tenido acceso a tanta información y conocimiento como en estos últimos siete años. Está demostrado que el uso adecuado de las TIC en los estudiantes ha permitido desarrollar su creatividad, descubrir y construir su propio aprendizaje de forma significativa, desarrollando sus destrezas a su propio ritmo, despertando su curiosidad y fomentando la investigación.

El uso de las TIC dentro del campo educativo cada vez ha ido adquiriendo más importancia, pero a partir de la crisis sanitaria se volvió protagonista en el proceso educativo porque fue la única herramienta que permitió el contacto de los docentes y estudiantes, exigiendo que los contenidos curriculares se adapten a esta nueva realidad $y$, dependiendo del nivel de conectividad del docente, de los estudiantes y de sus habilidades digitales, se podía transformar las clases rutinarias, [5], en clases dinámicas, novedosas y atractivas dando la oportunidad a los estudiantes de participar de forma activa y simultánea en su proceso de aprendizaje y construcción de su conocimiento.

Para lograr y aprovechar todas las bondades de la tecnología en el ámbito educativo, se requiere que todos los actores cuenten con un mínimo de herramientas para trabajar como, por ejemplo, un computador (de escritorio o portátil) o un teléfono inteligente, además de acceso a una red de internet. Y, lamentablemente, en este proceso que hemos vivido mundialmente, se ha hecho más evidente la brecha socioeconómica que hay, 
especialmente en el Ecuador, en el sector de San Juan de Calderón (por mencionar uno de los tantos sectores vulnerables) donde los estudiantes o bien no tienen acceso a internet, o bien carecen de equipos tecnológicos que les ayude a conectarse a sus clases. Otro punto desfavorable es que la realidad de las familias del sector, la mayoría de los representantes, prefieren que el tiempo destinado para las clases los hijos ayuden con labores dentro de casa, en el campo o en las ventas informales (actividad que las familias del sector, en su mayoría, se dedican). Este análisis da paso a la siguiente inquietud.

\section{Metodología}

Este artículo desea dar a conocer la problemática que existe en el sistema educativo en el ámbito tecnológico, debido a que es un aspecto que requiere mucha atención para evitar más deserción estudiantil y tomar en cuenta la realidad que estamos viviendo en nuestro país [8].

La investigación es de tipo exploratorio-descriptivo y la metodología de recolección de la información consistió en entrevistas a los diferentes actores: docentes, estudiantes y padres de familia, a una población de 3000 familias, se tomó una muestra de 559 personas. El área de estudio que se ha seleccionado es el barrio San Juan de Calderón (rural) y considerado sector vulnerable, por los altos índices de pobreza que se evidencia (Figura 1).

Familias de San Juan de Calderón

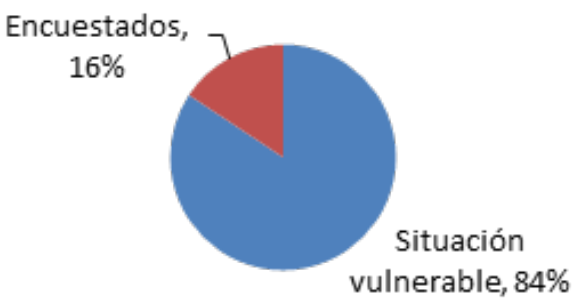

Figura 1. Familias de San Juan de Calderón

Los métodos utilizados en esta investigación son confiables, pues se realizó a través de una plataforma en la que los participantes, previo a la encuesta, dieron su aprobación para realizarla. Cabe recalcar que los métodos utilizados en esta investigación son validados por medio de la técnica de la encuesta, lo que determina que son datos confiables para dar verdadera importancia en el caso estudiado.

\section{Resultados}

El primer resultado que se obtuvo de la encuesta a los actores educativos de la comunidad de San Juan de Calderón fueron los siguientes: con respecto a la experiencia de los padres de familia y el uso de plataformas digitales, se puede visualizar en la figura 2.

1. Antes de la pandemia, ¿Tenía usted experiencia en el uso de plataformas digitales? (Zoom, Microsoft Teams, Google Classroom, Edmodo, Aula Virtual Moodle)

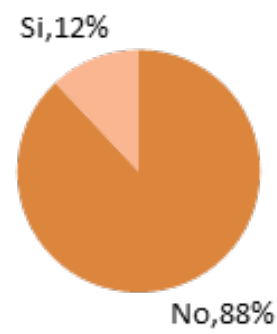

Figura 2. Experiencia de uso plataformas digitales

Este resultado es alarmante porque desde que se declaró la crisis sanitaria la comunicación se volvió $100 \%$ virtual y con el $88 \%$ de padres de familia sin ningún tipo de conocimiento tecnológico, poca era la ayuda que podían brindar a sus hijos, además que el nuevo currículo [9] eliminó la asignatura de Computación en su malla, lo que hizo más complicado la accesibilidad y manejo de las tecnologías a los estudiantes.

También se resaltó la brecha social que existe en nuestro país y a nivel mundial, de acuerdo a los datos de la ONU, que 70 millones de personas en el mundo viven en la pobreza extrema, en el Ecuador, lamentablemente tenemos varios sectores identificados como vulnerables, marcados por la pobreza y extrema pobreza. Podemos ver en la figura 3 que la mayoría de las familias cuenta con internet. Pero en la figura 4 se aclara la percepción de esta realidad, cuentan con internet, pero en su mayoría es de una red compartida por un familiar o un conocido, que les faci- 
lita la red por tiempo limitado (por ejemplo, solo en las horas de la mañana) para que se conecten a la clase y envíen deberes, pero para poder investigar y solventar inquietudes con sus docentes, ya no cuentan con el servicio de internet. Otra realidad es la de contar con internet, pero por medio de recargas de datos, (por ejemplo, \$ 1,00) [6] que le sirve para enviar fotos de las tareas de los estudiantes, pero no para conectarse a una clase o reunión (Figura 4).

\section{2. ¿Tiene acceso a internet en su hogar?}

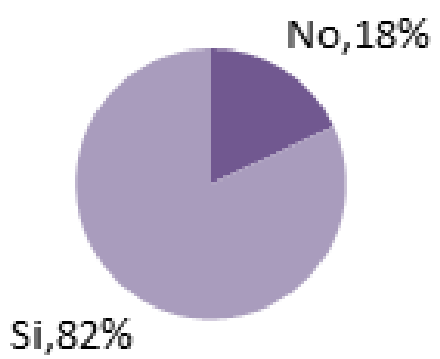

Figura 3. Acceso a internet

3. ¿Qué tipo de internet tiene?

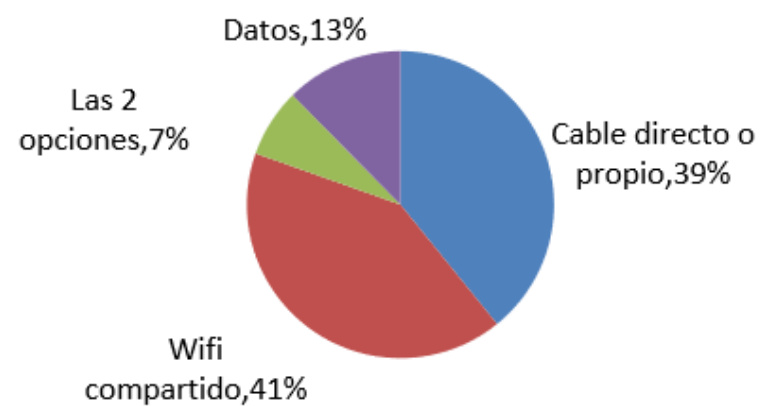

Figura 4. Tipo de internet

A estos datos se suman las dificultades de tener un equipo tecnológico adecuado para que los estudiantes puedan seguir sus clases, como lo menciono el economista Muhammad Yunus, ganador del premio Nobel de la Paz en el año 2006, [2] que el costo de los nuevos equipos tecnológicos solo provocan una brecha más amplia entre la tecnología y los sectores más pobres de la población mundial. Obviamente, porque una empresa siempre buscará tener ganancias y sus productos están direcciona- dos a una población con posibilidades económicas. Entonces, surge la inquietud, ¿la población pobre, no es parte de este mundo? Los datos recabados en la encuesta nos permitirán ver que un gran porcentaje de la familias cuentan con un medio tecnológico, pero muchas veces este recurso es el único en el hogar y en esta pandemia les tocó compartir entre los hijos (que tienen diferentes docentes y deben enviar diferentes evidencias de trabajo académico) y los padres de familia (quienes también debían usarlo para sus comunicaciones personales). Esto está en los gráficos 5 y 6

4.- ¿Tiene equipos tecnológicos en su hogar?

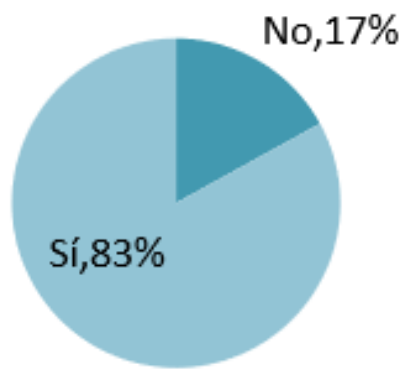

Figura 5. Equipos tecnológicos

\section{5. ¿Cuántos elementos tecnológicos tiene en su hogar?}

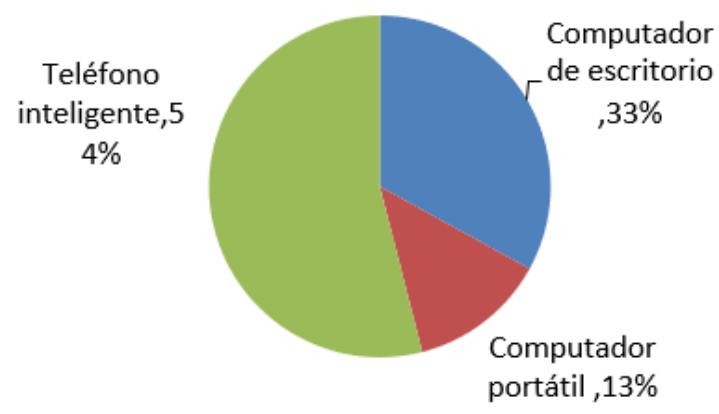

Figura 6. Cantidad de elementos tecnológicos

Esta dificultad en el acceso al internet, el tener un recurso tecnológico que permita a los estudiantes realizar con independencia sus actividades, es algo fuera de la realidad para este sector donde la mayoría de su población está dentro de los rangos de pobreza y pobreza extrema, que tienen un solo teléfono y en el mejor de los casos un computador para uso de todos los integrantes de la familia, a esto hay que sumar los aspectos que las familias 
comentaron que fueron difíciles enfrentar, por ejemplo, entender el funcionamiento de las plataformas digitales, escanear un documento, subir un documento en la aplicación de WhatsApp, etc. Lo que sí fue latente en las familias fue expresar su preocupación en el desarrollo pedagógico de sus hijos. Por las siguientes razones: primero su limitación económica; segundo, por el bajo nivel de escolaridad de los padres y madres de familia y no poder ayudar en sus dudas; $y$ tercero, que en comparación a las instituciones educativas privadas, la educación fiscal ha desmejorado notablemente durante esta pandemia.

7. Con las aplicaciones tecnológicas, ¿ Puede usted decir que, su hijo/os, en comparación a la educación presencial ha aprendido?

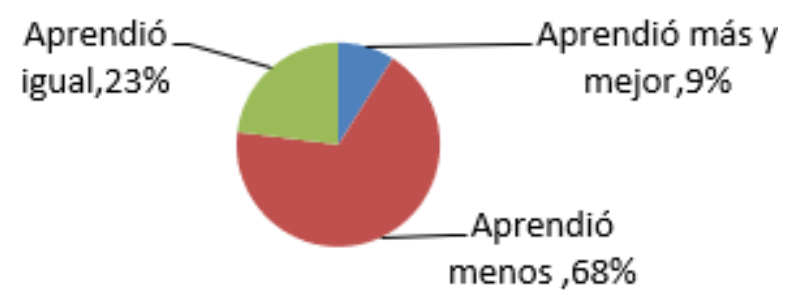

Figura 7. Aplicaciones tecnológicas

Esta dura realidad de varios sectores de la ciudad de Quito fue tan evidente que el día viernes 8 de enero del año 2021, el Ministerio de Educación hará la dotación de tablets para un número determinado de estudiantes de cinco instituciones educativas del sector de Calderón en general, [6] dentro de estas instituciones está considerada la unidad educativa que funciona en el sector de San Juan de Calderón.

Es admirable y loable esta acción del Ministerio de Educación, pero no es una solución definitiva porque la facilidad de conectarse, es decir, posee una red de internet, es otro de los aspectos que afecta a las familias. Pero es un logro y paso a paso el gobierno con los ministerios y subsecretarias deben seguir buscando alternativas de solución a este tema tan complejo que siempre ha existido, pero con esta pandemia se hizo más evidente.

\section{Conclusiones}

Se ha demostrado el impacto que han sufrido las familias en el manejo de la tecnología a causa de la pandemia, en especial en los sectores más vulnerables del Ecuador, nos ha permitido evidenciar aspectos importantes de la malla curricular que se debería retomar como, por ejemplo, la asignatura de Computación para los estudiantes de educación básica y bachillerato. También un llamado al Ministerio de Educación, Subsecretaría de Educación y Gobierno Central para dar verdaderas soluciones tecnológicas a los estudiantes de estos sectores de San Juan de Calderón que, como se mencionó, se entregarán tablets a algunos estudiantes de 5 instituciones de Calderón y Carapungo, pero falta solventar la conectividad, poder generar redes de wifi gratuitas, para que los estudiantes se puedan conectar a sus clases y realizar sus investigaciones.

Las TIC en la actualidad se han convertido en una herramienta fundamental en el proceso de enseñanza a nivel mundial, por lo tanto, es necesario contribuir en ella para que los estudiantes y padres de familias estén inmersa en el desarrollo personal, social y educativo.

Es importante manifestar que el uso de las TIC permite el desempeño competitivo de los educandos, es necesario en la actualidad tomar reto para mejorar el uso de ellas debido a que en estos momentos que estamos viviendo se ve difícil por el factor económico dentro de las familias, siendo este factor una desventaja en el aprendizaje de los estudiantes.

\section{Referencias}

[1] Gómez, J. R. (2004). Las TIC en educación. Catedrático de Filosofía en el IES Gabriel García Márquez de Madrid. Página Personal. Recuperado de http://www. ilustrados. com/ tema/13003/educacion. html.

[2] S. p. 1. i. y. b. vivir, «https://prezi.com/ivq8tv-fgttq/subsecretaria-para-la-innovacion-educativa-y-el-buen-vivir/?fallback $=1$,» 1404 2016. [En línea]. Available: https:// prezi.com/ivq8tv-fgttq/subsecretaria-para-la-innovacion-educativa-y-el-buen-vivir/?fallback=1. [Último acceso: 1208 2020]. 
[3] M. d. Gobierno, Plan Buen Vivir, Quito: Gobierno Central, 2010.

[4] E. comercio, «Quito es la ciudad que acumula más pérdidas económicas en la pandemia,» El comercio, p. 1, 2020.

[5] J. Carbonell, Pedagogías del Siglo XXI, Barcelona: Octaedro, 2015.

[6] B.B.C, «B.B.C,» 0111 2019. [En línea]. Available: bbc.com/mundo/noticias-america-latina-51990674. [Último acceso: 611 2020].
[7] . H. Cervantes Nieto, «OBLIGACIONES LABORALES DEL COVID 19,» Mexico, ISEF, 2019, pp. 5-19.

[8] C. A. Bernal Torres, «Metodología de la investigacion para la Admistracion, EconomÍa,Humanidades,Ciencias Sociales.,» 2da Edición ed., L. G. Figueróa, Ed., Mexico, 2006, p. 57.

[9] Mineduc, Currículo Preorizado para la emergencia., Quito, 2020-21. 